\title{
- An analytical method for estimation of parameters of Self Mixing Interferometric phase equation over all ${ }_{3}$ feedback regimes
}

\author{
Junaid Iqbal Khan \\ School of Electrical Engineering and Computer Sciences, National university of Sciences and \\ Technology, Islamabad, Pakistan \\ E-mail: jkhan.bee15seecs@seecs.edu.pk
}

\begin{abstract}
This paper presents a novel algorithm for measuring the linewidth enhancement factor of semiconductor lasers and the optical feedback level factor in a semiconductor laser with an external cavity. The proposed approach is based on analysis of the self-mixing phase equation to deduce equations for finding parameters given only knowledge of the perturbed phase. The effectiveness of the method has been validated with accuracy of $8.6 \%$ and $1.7 \%$ for $\mathrm{C}$ and $\alpha$ respectively while covering all feedback regimes.
\end{abstract}

\section{Keywords}

Linewidth enhancement factor (LEF), optical feedback, self-mixing interferometry, semiconductor lasers

\section{Introduction}

In optical feedback self-mixing interferometry(OFSMI), the instantaneous distance between the laser semiconductor diode driven by a constant injection current and a remote surface which back-scatters a small amount of optical power back into the laser diode cavity[7]. Linewidth enhancement factor (LEF) (denoted as $\alpha$ ) is a fundamental parameter of self-mixing interferometry as it characterizes the linewidth, the chirp, the injection lock range, and the response to optical feedback [5].The influence of the parameters $\mathrm{C}$ and $\alpha$ on the emitted laser intensity has been extensively analyzed in [1] and [2]. Establishing an accurate measurement has been a challenging and active research topic that has attracted extensive research work during the past two decades [5]. Existing approaches include the direct physical measurement of the subthreshold optical spectrum as the injected current is varied [3] and techniques based on the analysis of the locking regimes induced by optical injection from a master laser [4]. Moreover, an analytic method, based on gradient descent approach was presented [6], which showed the accuracy of $6.7 \%$ and $4.63 \%$ for $\mathrm{C}$ and respectively.

\section{Main theory}

When the optical feedback phenomenon occurs, the laser wavelength is no longer the constant $\lambda_{o}$ but is slightly modified and becomes a function of time $\lambda_{f}(t)$ when $\mathrm{D}(\mathrm{t})$ varies. The wavelength 
fluctuations can be found by solving the phase equation,

$$
x_{o}(t)=x_{f}(t)+C \sin \left(x_{f}(t)+\arctan (\alpha)\right)
$$

$x_{o}$ and $x_{f}$ are referred as perturbed and unperturbed phase respectively, $\alpha$ is the linewidth enhancement factor and $\mathrm{C}$ is the coupling factor. $x_{o}$ and $x_{f}$ can be represented as,

$$
\begin{aligned}
& x_{o}(t)=2 \pi \nu_{o}(t) \tau(t) \\
& x_{f}(t)=2 \pi \nu_{f}(t) \tau(t)
\end{aligned}
$$

where $\tau(t)=2 D(t) / c$ is the round trip time, with $\mathrm{c}$ as speed of light. $\nu_{f}(t)$ and $\nu_{o}(t)$ represents optical frequencies with and without feedback. Laser feedback output optical power(LDOOP) $\mathrm{P}(\mathrm{t})$ depends on the SM phenomenon and written as:

$$
P(t)=P_{o}\left[1+m \cos \left(x_{f}(t)\right)\right]
$$

where $P_{o}$ is the power emitted by the free-running state laser diode and $\mathrm{m}$ is the modulation index. Therefore, for purpose of displacement measurement, we track from SM signal $\mathrm{P}(\mathrm{t})$ measurement toward perturbed phase $x_{f}(t)$ to $x_{o}(t)$ toward displacement $\mathrm{D}(\mathrm{t})$ measurement.Due to environmental fluctuations and uncertainty, it is desirable to estimate parameters $\mathrm{C}$ and $\alpha$ for a given condition of the interferometer for robust displacement measurement.

\section{Theoretical derivation}

4.1. Estimation of alpha

Taking derivative on both sides of (1),

$$
\begin{gathered}
\frac{d x_{o}}{d t}=\frac{d x_{f}}{d t}\left(1+C \cos \left(x_{f}+\arctan (\alpha)\right)\right) \\
\frac{\frac{d x_{o}}{d x_{f}}}{d t}=1+C \cos \left(x_{f}+\arctan (\alpha)\right)
\end{gathered}
$$

When extremas of $x_{o}$ and $x_{f}$ are reached simultaneously, then their derivatives approach zero simultaneously and their ratio approaches 1 by taking limit on time,i.e, when $t=t_{\text {ext }}$,

$$
\lim _{t \rightarrow t_{e x t}} \frac{\frac{d x_{o}}{d t}}{\frac{d x_{f}}{d t}}=1
$$

Considering when $t=t_{e x t}$, then $x_{f}=x_{f e}$ and $x_{o}=x_{o e}$, Putting in (5),

$$
\begin{gathered}
\cos \left(x_{f e}+\arctan (\alpha)\right)=0 \\
x_{f e}+\arctan (\alpha)=k \pi-\frac{\pi}{2}
\end{gathered}
$$

Putting equation(8) in equation(1), as $\sin \left(k \pi-\frac{\pi}{2}\right)= \pm 1$

$$
x_{o e}=x_{f e} \pm C
$$

From equation(1), $x_{o}-x_{f}$ is maximally bounded by constant $\mathrm{C}$, when $x_{f}$ is allowed to increase from 0 to some value (less than $\frac{\pi}{2}-\arctan (\alpha)$ ). Therefore, on local maximas of displacement, $x_{o}$ swings $x_{f}$ by C. Lets denote maximas of $x_{o}$ and $x_{f}$ as $x_{o m}$ and $x_{f m}$ respectively,then on local maximas of displacement,

$$
x_{o m}=x_{f m}+C
$$

Page 2 of 8 
Putting in equation(1),

$$
\begin{gathered}
x_{f m}+C=x_{f m}+C \sin \left(x_{f m}+\arctan (\alpha)\right) \\
\sin \left(x_{f m}+\arctan (\alpha)\right)=1 \\
x_{f m}+\arctan (\alpha)=\frac{\pi}{2}+2 k \pi
\end{gathered}
$$

where, $\mathrm{k}$ is an integer.

$$
\alpha=\tan \left(\frac{\pi}{2}+2 k \pi-x_{f m}\right)
$$

Due to periodicity of tan function by $2 k \pi$, equation (12) becomes,

$$
\begin{gathered}
\alpha=\tan \left(\frac{\pi}{2}-x_{f m}\right) \\
\alpha=\tan \left(\arcsin (1)-x_{f m}\right)
\end{gathered}
$$

\subsection{Estimation of $C$}

Now, the perturbed phase $\left(x_{f}\right)$ being modified form of $x_{o}$ exhibits sharp transitions (from moderate to high feedback regime but it can be extended to all regimes once the algorithm is developed), then at those transitions derivative of $x_{f}$ approaches infinity. Let $x_{f}$ be represented as $x_{f t}$ on transitions of perturbed phase, then equation (5) becomes,

$$
\begin{gathered}
1+C \cos \left(x_{f t}+\arctan (\alpha)\right)=0 \\
C=\frac{-1}{\cos \left(x_{f t}+\arctan (\alpha)\right)}
\end{gathered}
$$

\section{Algorithm Design}

\subsection{Algorithm for alpha estimation}

Since derivative of $\arcsin (t)$ is given by,

$$
\frac{d(\arcsin (t))}{d t}=\frac{1}{\sqrt{1-t^{2}}}
$$

Around $\mathrm{t}=1, \frac{d \arcsin (t)}{d t}$ becomes quite large and sensitive, and slight deviations can lead to huge errors. Therefore, we can add counter sensitivity term as $\epsilon$ which has following properties.

$$
\epsilon<<1 \text { and } \epsilon \geq 0
$$

So, equation(14) becomes modified as,

$$
\alpha=\tan \left(\arcsin (1-\epsilon)-x_{f m}\right)
$$

42 If we are given information that $\alpha$ is bounded by some threshold ' $\tau \geq 0$ ' and 0 , then we find $\alpha$ 43 by following algorithm

Step 1: Initially choose $\epsilon=0$ and step size of varying it as $\delta \epsilon$

Step 2: For a given sample of perturbed phase $x_{f}$, find the maximum positive element as $x_{f m}$, $46 \quad$ which would be global maxima of $x_{f}(t)$.

Step 3: Plug the values into equation(21).If $\alpha$ is outside $[0, \tau]$, then increase $\epsilon$ to $\epsilon+\delta \epsilon$ and repeat $48 \quad$ Step 3.Otherwise, $\alpha$ would be required estimation. 


\subsection{Algorithm for $C$ estimation}

Due to sharpness of SM signal,a slight deviation of arguments of equation(18), can create huge errors. Due to this non-smooth property,we have to use statistic of finding globally sharpest transition and exploiting equation (18).

Step 1: For suitable magnitude threshold M, calculate derivative of sample of $x_{f}$ as $\frac{d x_{f}}{d t}$ and find the set $\mathrm{X}$ as,

$$
X=\left\{x_{f}(t) \mid t=\arg \left[\frac{d x_{f}}{d t} \geq M\right]\right\}
$$

Step 2: Plug the values into equation(18), then estimated value of $\mathrm{C}$ is,

$$
C=\max \left(\frac{-1}{\cos (x+\arctan (\alpha))}\right), \forall x \in X
$$

\section{Simulation Results}

6.1. Estimation of $C$ and alpha

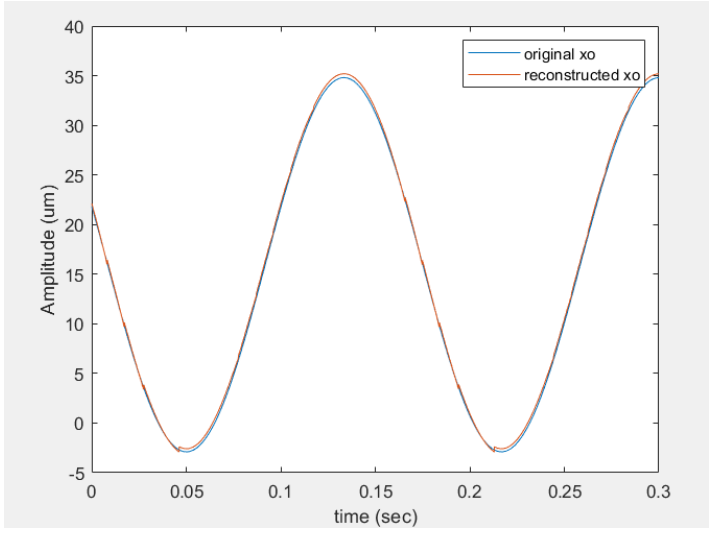

Figure 1. Reference unperturbed phase and reconstructed unperturbed phase for a displacement of $6 \mathrm{kHz}$

6.1.1. Remark The above-mentioned algorithm can be extended and conjugated with an optimization algorithm, as depicted in [6], which would decrease the number of iterations for convergence and increase accuracy. Moreover, an obvious observation is that an arbitrary smoothing digital filter could increase accuracy when reconstructing displacement.

\subsection{Reconstruction of unperturbed phase and displacement}

For a sinusoidal displacement of 3 peak to peak $\mu \mathrm{m}$ and $6 \mathrm{kHz}$, sampled at $10 \mathrm{kHz}$ with reference values of $\mathrm{C}$ and $\alpha$ as 4 and 5 respectively, on applying mentioned algorithms with $\tau=0.02$ and $M=10000$, we get a reconstructed unperturbed phase which gives mean square error of $32.4 \mathrm{~nm}$, by utilizing equation(1), as shown in figure(2) and (3) and reconstructed displacement is mentioned in figure(4). Displacement is reconstructed by using equation (2).

Similarly, for a sinusoidal displacement of 3 peak to peak $\mu \mathrm{m}$ and $6 \mathrm{kHz}, 12 \mathrm{kHz}$ and $18 \mathrm{kHz}$, sampled at $10 \mathrm{kHz}$ with reference values of $\mathrm{C}$ and $\alpha$ as 4 and 5 respectively, on applying mentioned algorithms with $\tau=0.19$ and $M=10000$, we get a mean square error of $96.1 \mathrm{~nm}$ and results are mentioned in figure(5), (6) and (7), after utilizing equation (1) and (2). To work with the mentioned method, the "feedback regimes" term would be ignored and values of $\mathrm{C}$ and $\alpha$ would be randomly experimented with, from 1 to 9 for $\mathrm{C}$ and 2 to 5 for $\alpha$ respectively. For simulation, a sample of the perturbed phase corresponding to a sinusoidal displacement of 3 peak to peak 


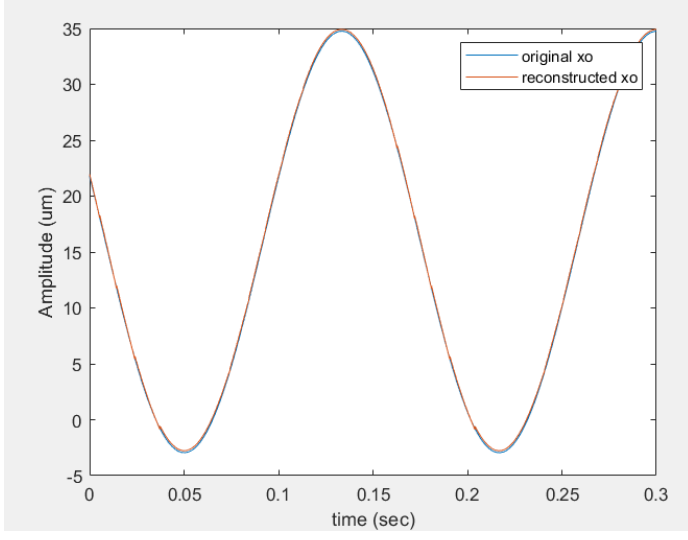

Figure 2. Plot of reference unperturbed phase and reconstructed unperturbed phase based on estimated $\mathrm{C}$ and $\alpha$ for a displacement of $6 \mathrm{kHz}$

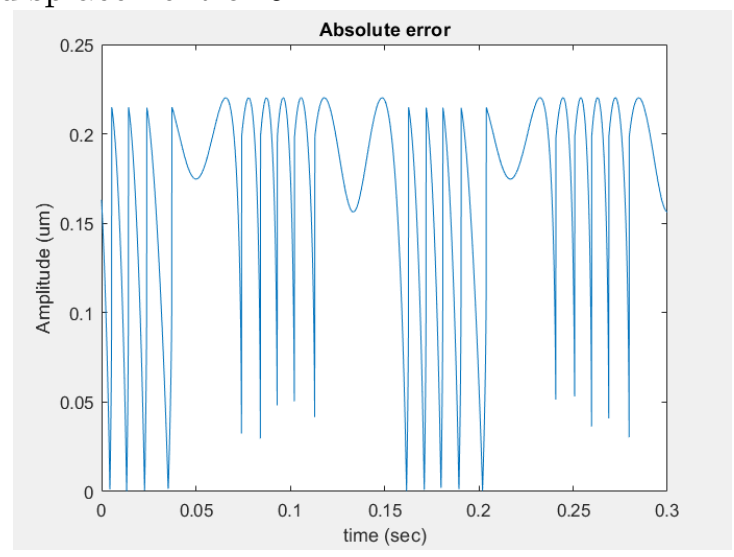

Figure 3. Plot of absolute error between reference unperturbed phase and reconstructed unperturbed phase based on estimated $\mathrm{C}$ and $\alpha$ for a displacement of $6 \mathrm{kHz}$

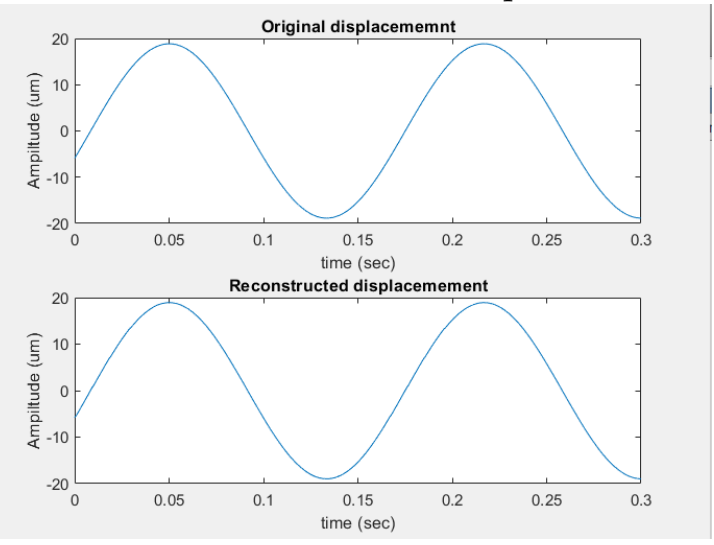

Figure 4. Reference displacement and reconstructed displacement for a displacement of $6 \mathrm{kHz}$ 


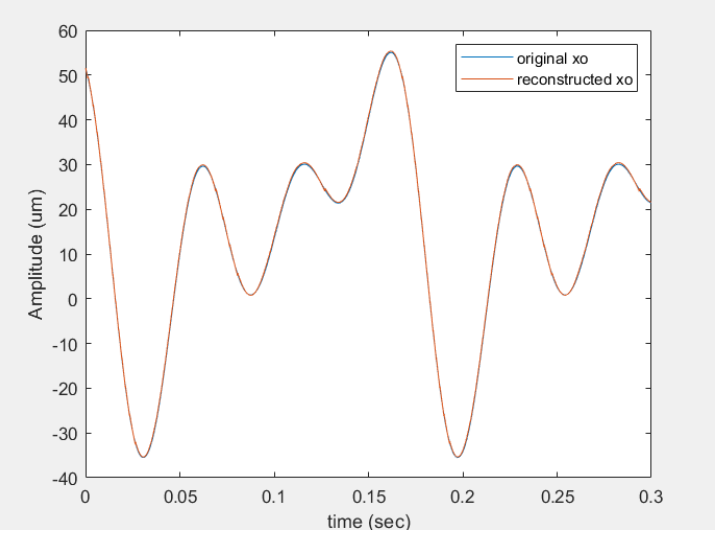

Figure 5. Plot of reference unperturbed phase and reconstructed unperturbed phase based on estimated $\mathrm{C}$ and $\alpha$ for a displacement having $6 \mathrm{kHz}, 12 \mathrm{kHz}$ and $18 \mathrm{kHz}$ components

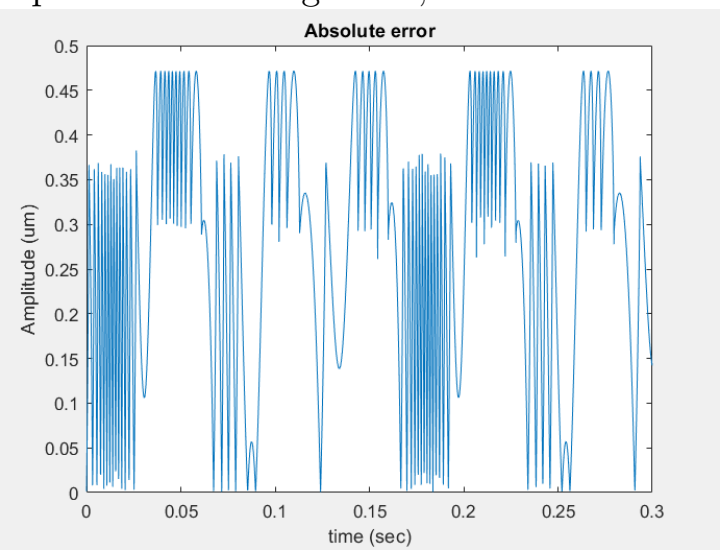

Figure 6. Plot of absolute error between reference unperturbed phase and reconstructed unperturbed phase based on estimated $\mathrm{C}$ and $\alpha$ for a displacement having $6 \mathrm{kHz}, 12 \mathrm{kHz}$ and $18 \mathrm{kHz}$ components
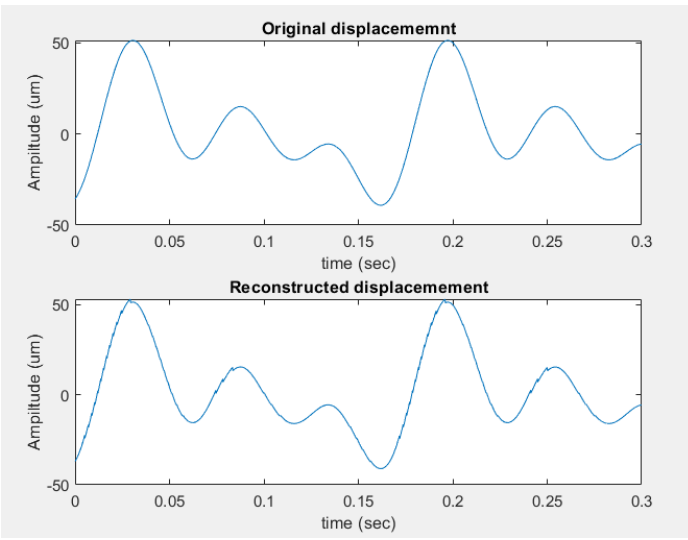

Figure 7. Reference displacement and reconstructed displacement for a displacement having $6 \mathrm{kHz}, 12 \mathrm{kHz}$ and $18 \mathrm{kHz}$ components 
$\mu \mathrm{m}$ and $6 \mathrm{kHz}$, sampled at $10 \mathrm{kHz}$, was used. The following table represents values of estimated values of $\mathrm{C}$ corresponding to reference $\mathrm{C}$ values, with fixed $\alpha=5$, along with $\tau=0.02$ and $M=10000$ found by hit and trial.

\begin{tabular}{|c|c|c|}
\hline Estimated $\mathrm{C}(\alpha=5)$ & Reference $\mathrm{C}(\alpha=5)$ & Percentage error \% \\
\hline 1.1708 & 1 & 17 \\
\hline 2.3401 & 2 & 17 \\
\hline 4.0779 & 4 & 1.9475 \\
\hline 5.4588 & 5 & 9.17 \\
\hline 6.6384 & 7 & 5.7 \\
\hline 9.1011 & 9 & 1.11 \\
\hline
\end{tabular}

The average error for above table is $8.67 \%$, for estimating $\mathrm{C}$ independently.

Similarly, following table presents estimated values of $\alpha$ corrosponding to reference values, when $\mathrm{C}$ is kept constant at 5

\begin{tabular}{|c|c|c|}
\hline Estimated $\alpha(\mathrm{C}=5)$ & Reference $\alpha(\mathrm{C}=5)$ & Percentage error $\%$ \\
\hline 2.1098 & 2 & 5.49 \\
\hline 3.0080 & 3 & 0.2667 \\
\hline 4.0013 & 4 & 0.0325 \\
\hline 5.0620 & 5 & 1.24 \\
\hline
\end{tabular}

The average error for above table is $1.75 \%$, for estimating $\alpha$ independently.

Simultaneous prediction of values of $\mathrm{C}$ and $\alpha$ corresponding to reference values are presented in following table.

\begin{tabular}{|c|c|c|}
\hline Estimated $\alpha, \mathrm{C}$ & Reference $\alpha, \mathrm{C}$ & Percentage error \% \\
\hline $3.4005,4.2041$ & 3,4 & $13.35,5.1$ \\
\hline $4.1603,5.1343$ & 3,5 & $38.6,2.6$ \\
\hline $5.2962,6.1946$ & 4,6 & $32.25,3.16$ \\
\hline $5.2674,7.1031$ & 5,7 & $5.2,1.42$ \\
\hline
\end{tabular}

The average error for estimating $\mathrm{C}$ and $\alpha$ simultaneously is $3.07 \%$ and $22.35 \%$ respectively. It should be noted that the above-presented data covers all feedback regimes.

Even though some errors appear large, for example, for reference $\mathrm{C}=6, \alpha=4$, give percentage errors of $3.16 \%$ and $32.25 \%$ respectively, when used for reconstruction of unperturbed phase from equation(1) gives means square error of $100 \mathrm{~nm}$, which is quite reasonable, and its depiction is presented in figure(1).

\section{Conclusion}

Therefore, based on SMI phase equation (1), the algorithm has been developed for estimation of $\mathrm{C}$ and $\alpha$ and is useful for reconstruction of unperturbed phase from sole knowledge of perturbed phase, for measurement of displacement of the target, under situations where $C$ and $\alpha$ are known or vary with the accuracy of $8.67 \%$ and $1.75 \%$ respectively, when independent estimation and $22.35 \%$ and $3.07 \%$ for simultaneous estimation. But even still, the mean square error bound for reconstructed displacement is less than $100 \mathrm{~nm}$.

\section{Acknowledgment}

This study was not funded.

\section{Conflict of Interest}

The author declares that he has no conflict of interest. 


\section{References}

[1] G Acket, Daan Lenstra, A Den Boef, and B Verbeek. The influence of feedback intensity on longitudinal mode properties and optical noise in index-guided semiconductor lasers. IEEE Journal of Quantum Electronics, 20(10):1163-1169, 1984.

[2] Silvano Donati, Guido Giuliani, and Sabina Merlo. Laser diode feedback interferometer for measurement of displacements without ambiguity. IEEE journal of quantum electronics, 31(1):113-119, 1995.

[3] Piotr Konrad Kondratko, Shun-Lien Chuang, Gabriel Walter, Theodore Chung, and Nick Holonyak Jr. Observations of near-zero linewidth enhancement factor in a quantum-well coupled quantum-dot laser. Applied physics letters, 83(23):4818-4820, 2003.

[4] G Liu, Xiaomin Jin, and Shun-Lien Chuang. Measurement of linewidth enhancement factor of semiconductor lasers using an injection-locking technique. IEEE photonics Technology letters, 13(5):430-432, 2001.

[5] Marek Osinski and Jens Buus. Linewidth broadening factor in semiconductor lasers-an overview. IEEE Journal of Quantum Electronics, 23(1):9-29, 1987.

[6] Jiangtao Xi, Yanguang Yu, Joe F Chicharo, and Thierry Bosch. Estimating the parameters of semiconductor lasers based on weak optical feedback self-mixing interferometry. IEEE Journal of Quantum Electronics, 41(8):1058-1064, 2005.

[7] Yanguang Yu, Yuanlong Fan, and Bin Liu. Self-mixing interferometry and its applications. In Optical Design and Testing VII, volume 10021, page 100210U. International Society for Optics and Photonics, 2016. 